\title{
Fibrés logarithmiques sur le plan projectif
}

\author{
Jean Vallès
}

16 juillet 2005

\begin{abstract}
Résumé : Nous décrivons le schéma des droites de saut des fibrés logarithmiques sur le plan projectif (thm 3.1]de ce texte). Connu, depuis l'article 2] de Dolgachev et Kapranov pour les fibrés de première classe de Chern paire, ce résultat est nouveau lorsque la première classe de Chern est impaire.
\end{abstract}

\begin{abstract}
We describe the scheme of jumping lines of logarithmic vector bundles on the projective plane (thm 3.1 of this text). This result is already proved by Dolgachev and Kapranov in [2] when the first Chern class is even, it is new when the first Chern class is odd.

"Ainsi les clartés, en s'accumulant, font figure d'enigmes, à la manière d'un verre trop épais qui cesse d'être transparent." (Simone Weil)
\end{abstract}

\section{Introduction}

Dolgachev et Kapranov ont étudié les fibrés logarithmiques, introduits par Deligne, dans le cas particulier où la base est un espace projectif complexe $\mathbb{P}^{n}$ et le diviseur associé une réunion d'hyperplans en position linéaire générale (voir les articles [1] et 2]). Ils montrent notamment que la donnée du diviseur détermine le fibré logarithmique associé (théorème 7.2 dit de "Torelli", voir aussi [5] pour une preuve différente).

Sur $\mathbb{P}^{2}$ les fibrés logarithmiques sont stables de rang deux. Selon que leur première classe de Chern est paire ou impaire, leur schéma de droites de saut est, en général, une courbe pour le cas pair, un schéma de longueur fini pour le cas impair. Dans les deux cas le degré de la courbe ou la longueur du schéma fini sont connus et donnés en fonctions des classes de Chern.

Puisque, d'après le théorème dit de "Torelli", le fibré est déterminé par la donnée du diviseur (ici une réunion de droites), ce diviseur doit aussi déterminer le schéma des droites de saut du fibré associé. Le lien est clairement établi dans [2] (voir thm 5.2) pour les fibrés de première classe de Chern paire via les courbes monoïdales mais il n'est pas fait pour les fibrés de première classe de Chern impaire. Plus précisément, bien que les droites du diviseur soient comptées avec multiplicité, elles ne suffisent pas à remplir le schéma de droites de saut. Il y a d'autres droites dans le schéma.

On se propose dans cet article, à partir d'un lien simple entre un fibré logarithmique sur le plan projectif et le faisceau d'idéaux du groupe de points associé dans le plan projectif dual, de dire qui sont ces droites supplémentaires (i.e nous donnons la description du schéma des droites de saut des fibrés logarithmiques de première classe de Chern impaire, théorème 3.1). Ainsi, de la même façon que dans le cas pair une unique courbe appelée 
courbe monoïdale était associée aux droites de départ (le diviseur du fibré logarithmique), dans le cas impair un autre ensemble fini de droites sera associé aux droites de départ.

L'étude des fibrés vectoriels stables via leur schéma des droites de saut est souvent ingrate dans le sens où les justifications géométriques (pourquoi cette droite saute t-elle et pas une autre?) n'apparaissent pas toujours. De ce point de vue l'intérêt des fibrés logarithmiques est que les justifications géométriques sont faciles à mettre en évidence. C'est ce que nous faisons dans la dernière partie en explicitant la géométrie sous-jacente du résultat principal de cet article.

\section{$1.1 \quad$ Notations}

On note $\mathbb{I} \subset \mathbb{P}^{2} \times \mathbb{P}^{2 \vee}$ la variété d'incidence points-droites de $\mathbb{P}^{2}, p$ et $q$ les projections $\mathbb{P}^{2} \stackrel{p}{\leftarrow} \mathbb{I} \stackrel{q}{\rightarrow} \mathbb{P}^{2 \vee}$.

On note $M_{\mathbb{P}^{2}}\left(c_{1}, c_{2}\right)$ l'espace de modules des fibrés vectoriels semi-stables sur $\mathbb{P}^{2}$ de classes de Chern $c_{1}$ et $c_{2}$. En général on préfèrera travailler avec les fibrés normalisés, c'est à dire dont la première classe de Chern vaut 0 ou -1 . On notera si nécéssaire $\mathcal{E}^{\text {norm }}$ le fibré normalisé correspondant à $\mathcal{E}$.

Étant donné un fibré stable $\mathcal{E}$ de rang deux on note $S(\mathcal{E})$ son schéma des droites de saut et $S_{\text {ens }}(\mathcal{E})$ l'ensemble de ses droites de saut.

\section{Lien entre sections globales et droites de saut}

Soit $F$ un fibré stable de rang deux sur $\mathbb{P}^{2}$ tel que $F \in M_{\mathbb{P}^{2}}(0,2 n-\epsilon)$ avec $\epsilon=0,1$. On rappelle qu'une droite $l \in \mathbb{P}^{2 \vee}$ est une droite de saut pour $F$ ssi $F_{l}=O_{l}(a) \oplus O_{l}(b)$ avec $|a-b|>1$. On suppose qu'il n'existe pas de droite $l$ telle que $H^{0} F_{l}(-2) \neq 0$ (cas général). L'idée principale est alors de comparer le fibré $F$ et le fibré $q_{*} p^{*} F$. Comme pour $l$ générale $F_{l}=O_{l} \oplus O_{l}$, on a une application

$$
\begin{gathered}
F \mapsto\left(q_{*} p^{*} F\right)^{\text {norm }} \in M_{\mathbb{P}^{2 \vee}}(0, n(n-1)) \quad \text { si } \epsilon=0 \\
F \mapsto\left(q_{*} p^{*} F\right)^{\text {norm }} \in M_{\mathbb{P}^{2 \vee}}\left(-1,(n-1)^{2}\right) \quad \text { si } \epsilon=1
\end{gathered}
$$

De plus le fibré $q_{*} p^{*} F$ est un fibré de Steiner (voir par exemple 2] pour la terminologie), plus précisément il s'inscrit dans une suite exacte

$$
0 \rightarrow q_{*} p^{*} F \longrightarrow H^{1}(F(-1)) \otimes O_{\mathbb{P}^{2 \vee}}(-1) \longrightarrow H^{1}(F) \otimes O_{\mathbb{P}^{2 \vee}} \rightarrow 0
$$

On a alors une première proposition (ensembliste) liant un fibré et son image directe.

\section{Proposition 2.1.}

$$
S_{\text {ens }}\left(q_{*} p^{*} F\right)=\left\{x \in \mathbb{P}^{2} \mid h^{0}\left(F \otimes \mathfrak{m}_{x}^{a}(a)\right) \neq 0 \text { pour } a<\left[\frac{2 n-\epsilon}{2}\right]\right\}
$$

Preuve de la proposition 2.1. On note $\mathbb{P}^{2}(x)$ l'éclatement de $\mathbb{P}^{2}$ le long d'un point $x \in \mathbb{P}^{2}$ et $p_{x}$ et $q_{x}$ les morphismes apparaissant dans le diagramme d'incidence

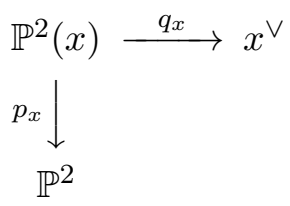


On vérifie facilement que l'absence de bisauteuse implique $q_{x *} p_{x}^{*} F=\left(q_{*} p^{*} F\right)_{\mid x^{\vee}}$.

Soit $x \in \mathbb{P}^{2}$, on suppose que la droite correspondante $x^{\vee}$ est une droite de saut de $q_{*} p^{*} F$. On a alors la décomposition $q_{*} p^{*} F_{\mid x^{\vee}}=O_{x \vee}(-a) \oplus O_{x \vee}(-b)$ avec $a+b=2 n-\epsilon$ et $a<\left[\frac{2 n-\epsilon}{2}\right]$. Comme $q_{x *} p_{x}^{*} F=\left(q_{*} p^{*} F\right)_{\mid x^{\vee}}$, cette décomposition fournit une section non nulle $h^{0}\left(p_{x}^{*} F \otimes q_{x}^{*} O_{x \vee}(a)\right) \neq 0$ ce qui équivaut à $h^{0}\left(F \otimes \mathfrak{m}_{x}^{a}(a)\right) \neq 0$.

Réciproquement, soit $s \in H^{0}\left(F \otimes \mathfrak{m}_{x}^{a}(a)\right)$ une section non nulle et $a<\left[\frac{2 n-\epsilon}{2}\right]$. Elle induit une suite exacte

$$
0 \rightarrow O_{\mathbb{P}^{2}} \longrightarrow F(a) \longrightarrow \mathcal{J}_{\Gamma}(2 a) \rightarrow 0
$$

où $\Gamma$ est un shéma de longueur finie vérifiant $\mathcal{J}_{\Gamma} \subset \mathfrak{m}_{x}^{a}$.

La section $p_{x}^{*} O_{\mathbb{P}^{2}} \longrightarrow p_{x}^{*} F(a)$ s'annule en codimension 1 le long de $a$-fois le diviseur exceptionnel $p^{-1}(x)$. Après simplification on obtient une section $q_{x}^{*} O_{x} \vee(-a) \longrightarrow p_{x}^{*} F$ ce qui prouve que $O_{x \vee}(-a) \hookrightarrow q_{*} p^{*} F_{\mid x \vee}$.

\section{Fibrés logarithmiques du plan}

Soit $Z$ un groupe de points de longueur $2 n+1-\epsilon$ en position linéaire générale de $\mathbb{P}^{2}$. C'est une conséquence du théorème 7.2 de [2] que le fibré logarithmique $E(Z)$ sur $\mathbb{P}^{2 \vee}$ associé à $Z$ est défini et entièrement déterminé par les données (a1) et (a2) :

a1) $E(Z)$ est un fibré de Steiner appartennant à $M_{\mathbb{P}^{2 \vee}}(0, n(n-1))$ si $\epsilon=0$, appartennant à $M_{\mathbb{P}^{2 \vee}}\left(-1,(n-1)^{2}\right)$ si $\epsilon=1$.

a2) Pour $x \in Z$ on a $E(Z)_{\mid x^{\vee}}=O_{x \vee} \oplus O_{x \vee}(2 n-\epsilon)$

Théorème 3.1. a) $E(Z)^{\vee}(-1)=q_{*} p^{*} \mathcal{J}_{Z}(1)$

b) Lorsque $\epsilon=0, S(E(Z))=\overline{\left\{x \in \mathbb{P}^{2}, h^{0}\left(\mathcal{J}_{Z} \otimes \mathfrak{m}_{x}^{n-1}(n)\right) \neq 0\right\}}$.

c) Lorsque $\epsilon=1, S(E(Z))$ est supporté par le lieu géométrique

$$
Z \cup \overline{\left\{x \in \mathbb{P}^{2}, h^{0}\left(\mathcal{J}_{Z} \otimes \mathfrak{m}_{x}^{n-2}(n-1)\right) \neq 0\right\}}
$$

et si $Z$ est général parmi les groupes de points du plan de longueur $2 n$ alors

$$
S(E(Z))=Z^{n-1} \sqcup\left\{x \in \mathbb{P}^{2}, h^{0}\left(\mathcal{J}_{Z} \otimes \mathfrak{m}_{x}^{n-2}(n-1)\right) \neq 0\right\}
$$

où $Z^{n-1}$ désigne le $(n-2)$-ième voisinage infinitésimal de $Z$ et $\sqcup$ l'union disjointe.

Remarque 1. La courbe $\overline{\left\{x \in \mathbb{P}^{2}, h^{0}\left(\mathcal{J}_{Z} \otimes \mathfrak{m}_{x}^{n-1}(n)\right) \neq 0\right\}}$ est notée $C(Z)$ par Dolgachev et Kapranov et est appelée complexe monoïdal associé à $Z$. Le point $b$ ) de ce théorème est déjà prouvé, avec une plus grande généralité, par ces deux auteurs (thm 5.2, [2]).

Remarque 2. Lorsque $Z$ est en position générale un simple calcul de dimension du système linéaire permet de vérifier que $S(E(Z)$ ) est fini lorsque $\epsilon=1$ (c'est pourquoi il n'est plus nécéssaire de prendre la clôture de Zariski de l'ensemble).

Remarque 3. Par contre lorsque $\epsilon=0$, l'irréductibilité de la courbe monoïdale associé à $Z$, même lorsque $Z$ est en position générale n'est pas établie (sauf pour $n \leq 3$ ).

Preuve du théorème 3.1. On considère une extension générale

$$
0 \rightarrow O_{\mathbb{P}^{2}} \longrightarrow F(1) \longrightarrow \mathcal{J}_{Z}(2) \rightarrow 0
$$


Comme $Z$ est en position linéaire générale il n'existe pas de trisécante à $Z$, ce qui équivaut au fait qu'il n'existe pas de droite $l$ telle que $h^{0} F_{l}(-2) \neq 0$. On en déduit alors que le fibré $q_{*} p^{*} F=q_{*} p^{*} \mathcal{J}_{Z}(1)$ est un fibré de Steiner avec les bonnes classes de Chern. Si $x \in Z$ il existe une section non nulle $s \in H^{0}\left(F \otimes \mathfrak{m}_{x}(1)\right)$, ce qui implique (comme dans la preuve de la proposition précédente) $q_{*} p^{*} F_{\mid x^{\vee}}=O_{x^{\vee}}(-1) \oplus O_{x^{\vee}}(2 n+1-\epsilon)$, ce qui prouve $\left.a\right)$.

Maintenant si $x \notin Z$ on a, pour tout entier $a$, une suite exacte

$$
0 \rightarrow \mathfrak{m}_{x}^{a}(a-1) \longrightarrow F \otimes \mathfrak{m}_{x}^{a}(a) \longrightarrow \mathcal{J}_{Z} \otimes \mathfrak{m}_{x}^{a}(a+1) \rightarrow 0
$$

De plus $H^{0} F \otimes \mathfrak{m}_{x}^{n-1}(n-1)=H^{0} \mathcal{J}_{Z} \otimes \mathfrak{m}_{x}^{n-1}(n)$ et $H^{0} F \otimes \mathfrak{m}_{x}^{n-2}(n-2)=H^{0} \mathcal{J}_{Z} \otimes \mathfrak{m}_{x}^{n-2}(n-1)$. Compte tenu de la proposition 2.1 ceci prouve $b$ ) et $c$ ).

Pour prouver le point $d$ ), nous calculons maintenant la longueur du sous-schéma de saut supporté par le diviseur $Z$.

Lemme 3.2. Soit $l$ une droite de saut d'un fibré $E \in M_{\mathbb{P}^{2}}(-1, n)$. Si $h^{0}\left(E_{l}(-k)\right)=1$ avec $k>0$ alors $S(E)$ contient le $(k-1)$-ième voisinage infinitésimal du point $l \in \mathbb{P}^{2 \vee}$.

Preuve du lemme 3.2. L'hypothèse fournit un homomorphisme surjectif de faisceaux

$$
E \longrightarrow O_{l}(-k-1)
$$

Notons $F(-1) \in M_{\mathbb{P}^{2}}(-2, n-k)$ le fibré noyau de cet homorphisme. En appliquant le foncteur $q_{*} p^{*}$ on trouve

$$
R^{1} q_{*} p^{*} F(-1) \longrightarrow R^{1} q_{*} p^{*} E \longrightarrow R^{1} q_{*} p^{*} O_{l}(-k-1) \rightarrow 0
$$

Tout d'abord, on rappelle que $R^{1} q_{*} p^{*} O_{l}(-k-1)=O_{\mathbb{P}^{2 \vee}} / \mathfrak{m}_{l}^{k}$ (il suffit, pour le prouver de prendre dans $\mathbb{P}^{2}$ une résolution de $O_{l}(-k-1)$ et d'utiliser $\left.R^{1} q_{*} p^{*} O_{\mathbb{P}^{2}}(-m)=S^{m-2}(\Omega(1))\right)$. La surjection

$$
R^{1} q_{*} p^{*} E \longrightarrow O_{\mathbb{P}^{2 \vee}} / \mathfrak{m}_{l}^{k} \rightarrow 0
$$

implique alors (voir [3] prop. B2 et B3 par exemple) que $S(E)$ contient le $(k-1)$-ième voisinage infinitésimal du point $l \in \mathbb{P}^{2 \vee}$. Enfin, si $l \notin \operatorname{supp}\left(R^{1} q_{*} p^{*} F(-1)\right)$ i.e. si $l$ n'est pas une droite de saut de $F$ alors le sous-schéma de $S(E)$ supporté par $l$ est exactement le $(k-1)$-ième voisinage infinitésimal de $l$ et il est de longueur $\left(\begin{array}{c}k+1 \\ 2\end{array}\right)$.

Revenons au cas des fibrés logarithmiques. Supposons donné $Z=\left\{l_{1}, \cdots, l_{2 n}\right\}$ notre groupe de points en position générale. On a une suite exacte canonique (par [2] prop. 2.9)

$$
0 \longrightarrow E\left(\left\{l_{1}, \cdots, l_{2 n-1}\right\}\right) \longrightarrow E\left(\left\{l_{1}, \cdots, l_{2 n}\right\}\right) \longrightarrow O_{l_{2 n}} \longrightarrow 0
$$

Comme la première classe de Chern de $E\left(\left\{l_{1}, \cdots, l_{2 n-1}\right\}\right)$ est paire son schéma de droites de saut est une courbe. Si l'on choisit $l_{2 n}$ hors du support de cette courbe, le sousschéma de $S\left(E\left(\left\{l_{1}, \cdots, l_{2 n}\right\}\right)\right)$ supporté par $l_{2 n}$ est exactement le $(n-2)$-ième voisinage infinitésimal de $l_{2 n}$. On en déduit que le sous-schéma supporté par $Z$ est exactement $Z^{n-1}$. De plus comme la la longueur du schéma de droites de saut de $E(Z)$ est égale à $\left(\begin{array}{c}(n-1)^{2} \\ 2\end{array}\right)$ et la longueur du sous-schéma supporté par $Z$ est $2 n \cdot\left(\begin{array}{c}n-1 \\ 2\end{array}\right)$, le schéma restant est de longueur $\frac{n(n-1)(n-2)(n-3)}{2}$. Ce nombre est justement le degré de la sous variété $\mathfrak{X}_{n-1, n-2} \subset \mathbb{P}\left(H^{0}\left(O_{\mathbb{P}^{2}}(n-1)\right)^{*}\right)$ des courbes de degré $(n-1)$ possèdant un point singulier 
d'ordre $(n-2)$ (ce que nous expliquerons plus en détail dans le paragraphe qui suivra l'exemple prototypique ci-dessous). Ceci prouve que la réunion est disjointe.

La proposition suivante explicite les liens entre les droites de saut de $E(Z)$ où $Z$ est un groupe de points de longueur $2 n$ en position linéaire générale et celles de $E(Z \cup\{x\})$ où $Z \cup\{x\}$ est en position linéaire générale.

Proposition 3.3. Soient $Z$ un groupe de points de longueur $2 n$ en position linéaire générale et $U_{Z} \subset \mathbb{P}^{2}$ l'ouvert des points $x$ tels que $Z \cup\{x\}$ soit en position linéaire générale. On a alors,

$$
S(E(Z)) \subset \bigcap_{x \in U_{Z}} S(E(Z \cup\{x\}))
$$

et surtout, lorsque $Z$ est général parmi les groupes de points du plan de longueur $2 n$, leurs supports coïncident.

Remarque : L'hypothèse supplémentaire sur $Z$ concernant l'égalité des supports n'est pas nécéssaire à mon avis mais, sans elle, je n'ai pas de preuve valable.

Preuve de la proposition [3.3. Soit $x \in U_{Z}$, on considère l'extension (d'après $a$ ) du théorème 3.1] par exemple ou bien [2] prop 2.9)

$$
0 \longrightarrow E(Z) \longrightarrow E(Z \cup\{x\}) \longrightarrow O_{x^{\vee}} \longrightarrow 0
$$

Toute droite de saut $l$ de $E(Z)$ est aussi une droite de saut de $E(Z \cup\{x\})$. En effet considérons la restriction de le suite exacte ci-dessus à la droite $l$ on obtient

$$
0 \longrightarrow O_{l}(n+1) \oplus O_{l}(n-2) \longrightarrow E(Z \cup\{x\}) \otimes O_{l} \longrightarrow O_{x^{\vee} \cap l} \longrightarrow 0
$$

L'injectivité de la première flèche implique que $E(Z \cup\{x\}) \otimes O_{l} \neq O_{l}(n) \oplus O_{l}(n)$ i.e que $l$ est une droite de saut pour $E(Z \cup\{x\})$, d'où

$$
S(E(Z)) \subset \bigcap_{x \in U_{Z}} S(E(Z \cup\{x\}))
$$

L'égalité des supports sera prouvée dans la proposition 4.2 du paragraphe qui suit.

Exemple. Soit $Z=\left\{l_{1}, \cdots, l_{8}\right\}$. Le fibré logarithmique $E(Z)$ après normalisation a pour classes de Chern $c_{1}=-1$ et $c_{2}=9$. Les droites de saut $l_{i}$ sont des bisauteuses et apparaissent avec une multiplicité égale à 3 (voir lemme 3.2), i.e la longueur du sous-schéma des droites de saut supporté par $Z$ est 24 . La longueur du schéma des droites de saut de $E(Z)$ est 36. Par ailleurs ce fibré étant déterminé par la donnée des huit bisauteuses de $Z$ les douze droites manquantes sont déterminées uniquement par la donnée de $Z$. Considérons le pinceau de cubiques passant par $Z$. Le lieu des points singuliers des cubiques du pinceau sont les droites de saut de $E(Z)$ distinctes de $Z$. Dans le $\mathbb{P}^{9}$ des cubiques du plan l'hypersurface des cubiques singulières, donné par le discriminant, est de degré 12 . Notre pinceau de cubiques rencontre cette hypersurface en douze points.

Il est difficile de parler de cubiques passant par huit points sans dire un mot du neuvième.

Remarque 3.4. Le pinceau de cubiques associé aux huit points en position générale de $Z=\left\{l_{1}, \cdots, l_{8}\right\}$ définit un neuvième point $l_{9}$. La droite correspondante n'est pas une droite de saut de $E(Z)$. 
En effet, si une cubique du pinceau admet ce neuvième point comme point singulier elle intersecte la cubique générale du pinceau en degré $\geq 10$. Par conséquent la cubique générale est décomposée ce qui contredit l'hypothèse de position générale.

Les cas limites sont les suivants : soit $C$ une conique lisse. Si $Z \in C$ alors $E(Z)$ est un fibré de Schwarzenberger et son schéma de droites de saut est le diviseur $S(E(Z))=3 C$ (voir [4, prop 2.1). Si $Z \backslash\left\{l_{8}\right\} \in C$, alors $S\left(E(Z)\right.$ ) est supporté par $C \cup\left\{l_{8}\right\}$.

\section{Surfaces rationnelles, dualité et droites de saut}

A partir d'un groupe de points plan de longueur $2 n$, on retrouve par des procédés classiques (éclatement, projections, variétés tangentes...) les courbes monoïdales associées (en ajoutant un point) ainsi que le groupe de points supplémentaire. Avant de détailler cette construction j'en donne ci-dessous la trame.

\subsection{Squelette de la construction}

Soit $Z_{0}$ un groupe de points plan en position générale de longueur $2 n$. Les courbes de degré $n$ avec un point singulier d'ordre $(n-1)$ passant par $Z_{0}$ forment, dans l'espace projectif des courbes de degré $n$ passant par $Z_{0}$, une surface que nous noterons $S_{1}$. Une section hyperplane de cette surface par un hyperplan $(n-1)$-tangent en un point $x$ est une courbe birationnelle à la courbe monoïdale $C\left(Z_{0} \cup\{x\}\right)$.

Comme les courbes $C_{i}$ de degré $(n-1)$ avec un point singulier $x_{i}$ d'ordre $(n-2)$ passant par $Z_{0}$ sont en nombre fini, égal à $N$, la surface $S_{1}$ contient $N$ droites qui se contractent en un groupe de points $\Gamma=\left\{x_{1}, \cdots, x_{N}\right\}$ sur $\mathbb{P}^{2}$. En effet, étant donné une courbe $C_{i}$ de degré $(n-1)$ passant par $Z_{0}$ qui est $(n-2)$-singulière en un point $x_{i}$, le $\mathbb{P}^{1}$ de courbes de degré $n$ obtenues en prenant la réunion de $C_{i}$ et d'une droite passant par $x_{i}$ est bien contenu dans $S_{1}$. Il en résulte que toutes les courbes monoïdales de la forme $C\left(Z_{0} \cup\{x\}\right)$ ont $\Gamma$ pour lieu base. On obtient alors $S\left(E\left(Z_{0}\right)\right)=Z_{0}^{n-1} \cup \Gamma$.

\subsection{Détails de la construction}

Soit $V$ un espace vectoriel complexe de dimension 3. On note $\mathbb{P}^{2}=\mathbb{P} V$ et $H^{0}\left(O_{\mathbb{P}^{2}}(1)\right)=V$. Considérons l'application canonique de dérivation (application duale de la multiplication)

$$
\partial_{n-2}: S^{n} V \rightarrow S^{n-2} V \otimes S^{2} V
$$

elle induit l'homomorphisme suivant de fibrés vectoriels

$$
0 \longrightarrow S^{n-2} V^{*} \otimes O_{\mathbb{P}^{2}}(-2) \stackrel{\partial_{n-2}}{\longrightarrow} S^{n} V^{*} \otimes O_{\mathbb{P}^{2}} \longrightarrow E_{n-1} \longrightarrow 0
$$

où la matrice $\partial_{n-2}$ est formée des dérivées partielles $(n-2)$-ièmes d'une base de $S^{n} V^{*}$. Le fibré projectif

$$
\mathbb{P}\left(E_{n-1}\right) \subset \mathbb{P}^{2} \times \mathbb{P}\left(S^{n} V^{*}\right)
$$

est décrit par l'incidence $\left\{(x, f) ; \partial_{n-2} f(x)=0\right\}$. La fibre au dessus d'un point $x \in \mathbb{P}^{2}$ s'identifie à l'espace projectif $\mathbb{P}\left(H^{0}\left(\mathfrak{m}_{x}^{n-1}(n)\right)\right)$ où $\mathfrak{m}_{x}$ est le faisceau d'idéaux du point $x$. Son image $\mathfrak{X}_{n, n-1}$ dans $\mathbb{P}\left(S^{n} V^{*}\right)$ par la seconde projection est la variété des courbes de 
degré $n$ de $\mathbb{P}^{2}$ qui s'annulent à l'ordre $n-1$ en un point de $\mathbb{P}^{2}$, autrement dit les hyperplans $(n-1)$-tangents à la surface de Veronese $v_{n}(\mathbb{P} V) \subset \mathbb{P} S^{n} V$. Le fibré projectif est birationnel à son image $\mathfrak{X}_{n, n-1}$. La dimension, égale à $2 n+2$, de $\mathfrak{X}_{n, n-1}$ ainsi que son degré, égal à $\frac{(n+1) n(n-1)(n-2)}{2}$, se déduisent des rang et classes de Chern du fibré $E_{n, n-1}$.

\section{La surface $S_{1}$}

Soit $Z_{0}$ un groupe de points de $\mathbb{P}^{2}$ en position générale et de longueur $2 n$. On considère la surface $S_{1}=\mathfrak{X}_{n, n-1} \cap \mathbb{P}\left(H^{0}\left(J_{Z_{0}}(n)\right)^{*}\right)$. C'est une surface rationnelle provenant de l'éclatement de $\mathbb{P}^{2}$ le long d'un schéma de longueur finie $Z_{1}$ défini par $Z_{0}$ que nous explicitons ci-dessous.

La restriction $\left(S^{n} V\right)^{*} \stackrel{r e s}{\longrightarrow} H^{0}\left(J_{Z_{0}}(n)\right)^{*}$ composée avec l'homorphisme $\partial_{n-2}$ induit une suite exacte

$$
0 \longrightarrow S^{n-2} V^{*} \otimes O_{\mathbb{P}^{2}}(-2) \stackrel{M}{\longrightarrow} H^{0}\left(J_{Z_{0}}(n)\right)^{*} \otimes O_{\mathbb{P}^{2}} \longrightarrow J_{Z_{1}}(n(n-1)) \longrightarrow 0
$$

où le schéma de longueur finie $Z_{1}$ est le lieu base du système linéaire défini par les mineurs maximaux de la matrice $M$. Le support de $Z_{1}$ est formé des points $x_{1}$ du plan pour lesquels $h^{0}\left(J_{Z_{0}} \otimes \mathfrak{m}_{x_{1}}^{n-1}(n)\right) \geq 2$. C'est le cas par exemple lorsque $x_{1} \in Z_{0}$, ce qui montre que $Z_{0} \subset Z_{1}$. La surface $S_{1}$ est l'image de l'éclaté de $\mathbb{P}^{2}$ le long de $Z_{1}$ par le morphisme composé :

$$
\mathbb{P}\left(J_{Z_{1}}(n(n-1))\right) \hookrightarrow \mathbb{P} V \times \mathbb{P}\left(H^{0}\left(J_{Z_{0}}(n)\right)^{*}\right) \stackrel{p r_{2}}{\rightarrow} \mathbb{P}\left(H^{0}\left(J_{Z_{0}}(n)\right)^{*}\right)
$$

\section{Les courbes monoïdales}

Etant donné $x \in \mathbb{P}^{2} \backslash Z_{0}$, le conoyau de l'application composée

$$
S^{n-2} V^{*} \otimes O_{\mathbb{P}^{2}}(-2) \rightarrow H^{0}\left(J_{Z_{0}}(n)\right)^{*} \otimes O_{\mathbb{P}^{2}} \rightarrow H^{0}\left(J_{Z_{0} \cup\{x\}}(n)\right)^{*} \otimes O_{\mathbb{P}^{2}}
$$

est supporté par la courbe monoïdale $C\left(Z_{0} \cup\{x\}\right)$. Cette courbe est une section de $J_{Z_{1}}(n(n-1))$ birationnelle à la courbe d'intersection $\mathfrak{X}_{n, n-1} \cap \mathbb{P}\left(H^{0}\left(J_{Z_{0} \cup\{x\}}(n)\right)^{*}\right)$, elle est donnée ensemblistement par $\left\{y \in \mathbb{P}^{2} \mid h^{0}\left(J_{Z_{0} \cup\{x\}} \otimes \mathfrak{m}_{y}^{n-1}(n)\right) \neq 0\right\}$.

\section{Le groupe de point éclaté}

On voudrait maintenant donner une description plus précise du groupe de points $Z_{1}$. Compte tenu de ce que nous avons vu ci-dessus, aux points de $Z_{1}$ il apparaît au moins un $\mathbb{P}^{1}$ de courbes de degré $n$ qui sont $(n-1)$-singulières. On veut montrer que cette situation (pour $Z_{0}$ en position générale) est entièrement décrite par l'union d'une courbe de degré $(n-1)$ ayant un point singulier d'ordre $(n-2)$ et d'une droite passant par ce point singulier.

Lemme 4.1. Soit $x \in \mathbb{P}^{2} \backslash Z_{0}$ un point tel que $h^{0}\left(J_{Z_{0}} \otimes \mathfrak{m}_{x}^{n-1}(n)\right) \geq 2$. Alors une section générale de $H^{0}\left(J_{Z_{0}} \otimes \mathfrak{m}_{x}^{n-1}(n)\right)$ est la réunion d'une courbe de degré $(n-1)$ qui est $(n-2)$ singulière en $x$ et d'une droite passant par $x$.

Preuve du lemme 4.1. Un pinceau de courbes singulières possède une composante commune. Considérons deux courbes du pinceau. Si elles n'ont pas de composante commune elles se coupent le long d'un schéma de longueur $n^{2}$ qui contient un sous-schéma de longueur au moins $(n-1)^{2}$ au point base singulier. Ceci implique qu'en dehors du point singulier elles doivent se couper au plus le long de $2 n-1$ points, ce qui contredit l'hypothèse. Montrons que cette composante est une courbe de $\mathfrak{X}_{n-1, n-2}$ singulière en $x$. 
Comme $Z_{0}$ est en position générale le lieu $\left\{x \in \mathbb{P}^{2}, h^{0}\left(\mathcal{J}_{Z_{0}} \otimes \mathfrak{m}_{x}^{n-3}(n-2)\right) \neq 0\right\}$ est vide et le lieu $\left\{x \in \mathbb{P}^{2}, h^{0}\left(\mathcal{J}_{Z_{0}} \otimes \mathfrak{m}_{x}^{n-2}(n-1)\right) \neq 0\right\}$ est un schéma de longueur finie. Par conséquent une courbe de $S_{1}$ (c'est à dire une courbe de $\mathbb{P}^{2}$ correspondante à un point de $S_{1}$ ) qui n'est pas irréductible est obligatoirement de la forme $C \times L$ avec $C \in$ $\mathfrak{X}_{n-1, n-2} \cap \mathbb{P}\left(H^{0}\left(J_{Z_{0}}(n-1)\right)^{*}\right)$ et $L$ une droite passant par $x$.

Comme $\mathfrak{X}_{n-1, n-2} \cap \mathbb{P}\left(H^{0}\left(J_{Z_{0}}(n-1)\right)^{*}\right)$ est un groupe de points de longeur $N=\operatorname{deg} \mathfrak{X}_{n-1, n-2}$ (les courbes $C_{i}$ qui sont $(n-2)$-singulières aux points $\left.x_{i}\right)$ la surface $S_{1}=\mathfrak{X}_{n, n-1} \cap$ $\mathbb{P}\left(H^{0}\left(J_{Z_{0}}(n)\right)^{*}\right)$ contient alors $\operatorname{deg}\left(\mathfrak{X}_{n-1, n-2}\right)$ droites. Ces droites sont contractées sur $\mathbb{P}^{2}$ (le même point singulier pour toute la droite). On note $\Gamma=\left\{x_{1}, \cdots, x_{N}\right\}$ le groupe de points image dans $\mathbb{P}^{2}$.

Proposition 4.2. Le support de $\bigcap_{x \in U_{Z_{0}}} C\left(Z_{0} \cup\{x\}\right), Z_{1}$ et de $S\left(E\left(Z_{0}\right)\right)$ est $Z_{0} \cup \Gamma$. Plus précisément, $Z_{1}=\tilde{Z}_{0} \cup \Gamma$ et $S\left(E\left(Z_{0}\right)\right)=Z_{0}^{n-1} \cup \Gamma$ où $\tilde{Z}_{0}$ est supporté par $Z_{0}$ et est localement intersection complète $(n-1) \times(n-1)$.

Preuve de la proposition 4.2. D'après le lemme précédent $Z_{1}$ est ensemblistement la réunion de $Z_{0}$ et de $\Gamma$. Par ailleurs lorsque $Z_{0}$ est en position générale les courbes monoïdales $C\left(Z_{0} \cup\{x\}\right)$ engendrent l'espace $\mathbb{P}\left(H^{0} J_{Z_{1}}(n(n-1))\right)$. Autrement dit $Z_{1}=$ $\bigcap_{x \in U_{Z_{0}}} C\left(Z_{0} \cup\{x\}\right)$. On en déduit que $S\left(E\left(Z_{0}\right)\right) \subset Z_{1}$ ou encore que $Z_{1}$ contient le $(n-2)$-ième voisinage infinitésimal de $Z_{0}$. Comme $Z_{1}$ est localement intersection complète (c'est le lieu d'annulation d'une section d'un fibré vectoriel de rang deux!) et comme $l\left(O_{Z_{1}}\right)=(n-1)^{2} l\left(O_{Z_{0}}\right)+\operatorname{deg}\left(\mathfrak{X}_{n-1, n-2}\right)$ on en déduit le résultat.

\section{Références}

[1] P.Deligne, Théorie de Hodge II, Publ.Math. IHES, 40 (1971), 5-58.

[2] I.Dolgachev et M.Kapranov, Arrangements of hyperplanes and vector bundles on $\mathbf{P}_{n}$, Duke Math.J. 71, (1993), 633-664.

[3] L. Gruson et C. Peskine, Courbes de l'espace projectif : variétés de sécantes, Progress in Math 24 (1982)

[4] Vallès, J., Conique de droites de saut et Fibrés de Schwarzenberger, BSMF, (2000).

[5] Vallès, J., Nombre maximal d'hyperplans instables pour un fibré de Steiner, Math. Zeit., 233, (2000), 507-514. 